\title{
Measurement and Simulation Based Study on UWB Channel Characteristics on the Abdomen Area
}

\author{
Mariella Särestöniemi \\ Center for Wireless Communications, \\ Faculty of Information Technology and \\ Electrical Engineering \\ University of Oulu \\ Oulu, Finland \\ mariella.sarestoniemi@oulu.fi \\ Timo Kumpuniemi \\ Center for Wireless Communications, \\ Faculty of Information Technology and \\ Electrical Engineering \\ University of Oulu \\ Oulu, Finland \\ Timo.Kumpuniemi@oulu.fi \\ Matti Hämäläinen \\ Center for Wireless Communications, \\ Faculty of Information Technology and \\ Electrical Engineering \\ University of Oulu \\ Oulu, Finland \\ matti.hamalainen@oulu.fi
}

\author{
Chaïmaâ Kissi \\ Electronics and Telecommunication \\ Systems Research Group, National \\ School of Applied Sciences (ENSA) \\ Ibn Tofail University \\ Kenitra, Morocco \\ chaimaakissi1@gmail.com \\ Marko Sonkki \\ Center for Wireless Communications, \\ Faculty of Information Technology and \\ Electrical Engineering \\ University of Oulu \\ Oulu, Finland \\ marko.sonkki@oulu.fi \\ Jari Iinatti \\ Center for Wireless Communications, \\ Faculty of Information Technology and \\ Electrical Engineering \\ University of Oulu \\ Oulu, Finland \\ jari.iinatti@oulu.fi
}

\author{
Carlos Pomalaza Raez \\ Department of Electrical and Computer \\ Engineering \\ Purdue University \\ Fort Wayne, Indiana 46805, USA \\ cpomalaz@purdue.edu \\ Sami Myllymäki \\ Microelectronics Research Unit, \\ Faculty of Information Technology and \\ Electrical Engineering \\ University of Oulu \\ Oulu, Finland \\ sami.myllymaki@oulu.fi
}

\begin{abstract}
This paper presents a comprehensive simulation and measurement data based study on the UWB on-body radio channel characteristics on the human abdomen area. The main target is to evaluate channel characteristics with several different antenna location options using a recently published ultra wideband (UWB) antenna designed for inbody communications, e.g. for capsule endoscope localization. For the simulations, we use two voxel models and two layer models. Measurement data consists of channel data for seven volunteers having different body size and body composition. The simulation and measurement data is compared and the reason for the differences is discussed. Furthermore, it is shown that the on-body channel characteristics vary significantly depending on the body size and shape of the volunteer if the antenna separation distance is large. With smaller antenna distances, the difference is minor.
\end{abstract}

Keywords-capsule endoscopy, radio channel, ulta wideband, wireless body area networks

\section{INTRODUCTION}

Recently, ultra wideband channel characteristics on the abdomen area has been an intensively studied topic [1]-[9]. This is due to the increasing interest on the development of medical monitoring devices, such as wireless capsule endoscope (WCE) [3]. Several capsule localization techniques have been presented in the literature [10]-[11]. All of them are based on the antenna groups. Locations of the antennas on the abdomen area plays as crucial role, especially if we would like to minimize the number of antennas.

There are two criteria to fulfill when determining antenna location on the abdomen area: there should be good "view" towards the small intestine so that antenna's main lobe is directed towards small intestine. Besides the propagation channel between the node antenna and the antenna of the on-body device should be strong enough that information obtained from the node antenna is received correctly. These criteria are taken into account when designing the antennas for WCE localization.

Channel characteristics on the abdomen area may vary significantly depending on the antennas type and for which purpose it has been designed. Channel studies using antennas designed for inbody communication are presented e.g. in [1]-[5]. The studies are based on voxel model or layer model simulations. In [7], we presented a simulation study on the UWB channel characteristics on the abdomen area with an on-body antenna designed for intra-body communications [12].

In this paper we continue the study of [7] by presenting measurement data based channel characteristics for the antenna locations of [7] as well as for two new antenna locations. The presented results consist of channel measurement data for seven volunteers having different body size and shape. Furthermore, we present simulation results using two voxel models and two layer models, and compare them with the measurement results. Up to our knowledge, this paper presents for the first time such a comprehensive study on the on-body channel characteristics with several simulation models and measurement data with several volunteers.

This paper is organized as follows: Section II presents the study case explaining the antennas, simulation and measurement setups, as well as antenna location options. Comparison of the simulation models is presented in Section III. Measurement results are presented and 
compared with the simulation results in Section IV. Conclusion and future work are discussed in Section V.

\section{STUDY CASE}

\section{A. Low band UWB Antenna}

In this study case, we used a recently published UWB onbody antenna, designed for inbody communications [12]. The antenna design is illustrated in Figure 1a. Besides, the prototype of the antenna is presented in Fig. 1b. The antenna itself is originally omnidirectional but with a cavity it becomes highly directional, which makes it suitable e.g. for WCE localization. The antenna is designed to work at frequency band $3.75-4.25 \mathrm{GHz}$, which meets the IEEE 802.15.6 standards requirements. A detail description of the antenna, antenna properties, and the impact of the cavity can be found in [12]. Furthermore, the free-space propagation studies as well as on-body antenna matching results with the studied antenna can be found in [13].
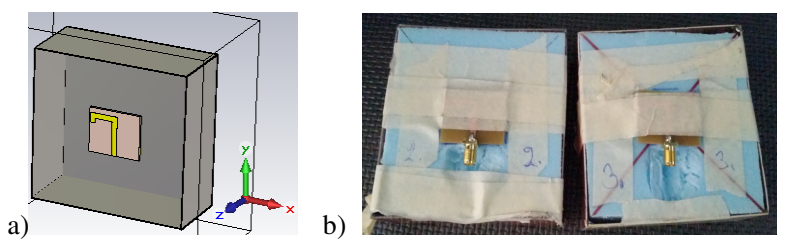

Figure 1. Low-band UWB cavity-backed on-body antenna: a) a designed model, b) prototypes used in the measurements.

\section{B. Simulations}

This paper presents similar simulation cases as presented [7] though they are modified slightly to resemble more the practical measurement cases. For instance, the coaxial cables were modeled on the antennas, since the cables can also have impact on the channel characteristics. Besides, the antennas are tilted along the body surface, similarly to measurement scenario. In the measurements, it was noted that the antenna's tilt angle may vary depending on the shape of the abdomen of the volunteer. However, in the simulations we used such a tilt that the antenna follows smoothly the skin surface.

The simulations were carried out using the electromagnetic propagation simulation software CST [14], which is based on finite integration technique. For the simulations, we selected Laura and Gustav voxel models from CST's voxel library. We included only the abdomen area and its surroundings in the simulations to reduce the simulation time remarkably. Torsos of Laura and Gustav voxels are presented in Fig.s $2 \mathrm{a}$ and $2 \mathrm{~b}$, respectively. Resolution of Laura voxel is $1.875 \mathrm{~mm} \times 1.875 \mathrm{~mm} \times 1.25$ $\mathrm{mm}$, whereas Gustav voxel has $2.08 \mathrm{~mm} \times 2.08 \mathrm{~mm} \times 2 \mathrm{~mm}$ resolution. The antennas were placed on the voxel models abdomen so that the distance between the outermost pixel of the voxel's skin and the coaxial cable used for the antenna feeding is $0 \mathrm{~mm}$, as shown in Fig. 2c. The distance between the outermost part of the coaxial cable and the antenna radiator (presented as a red spot in Fig. 2) is $0.366 \mathrm{~mm}$.

Furthermore, we evaluated our study cases with multilayer models of the abdomen area. The first multilayer model is planar, as presented in Fig. 3a. The second multilayer model is bended version of the first one, and is presented in Fig. 3b. Thicknesses of the tissue layers and dielectric properties of the tissues are presented in Table I.

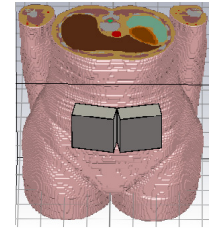

(a) (b)

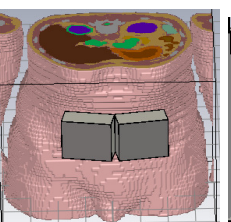

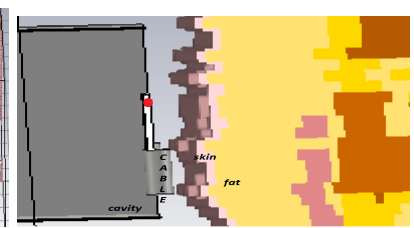

(c)
Figure 2. a) Laura voxel and b) Gustav voxel, c) antenna on Laura voxel.

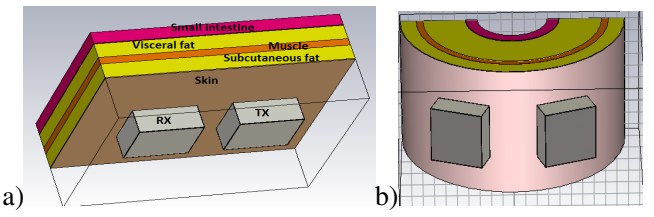

Figure 3. a) Planar multilayer model and b) bended multilayer model of the abdomen area for antenna location options 1 and 3, respectively.

TABLE I: Thickness of the layers and dielectric properties of tissues.

\begin{tabular}{|l|l|l|l|}
\hline Tissue & $\begin{array}{l}\text { Thickness } \\
(\mathrm{mm})\end{array}$ & $\begin{array}{l}\text { Permit- } \\
\text { tivity }\end{array}$ & $\begin{array}{l}\text { Conductivity } \\
(\mathrm{S} / \mathrm{m})\end{array}$ \\
\hline Skin & 1.4 & 40.85 & 2.701 \\
\hline Subcutaneous fat & 20 & 5.125 & 0.1829 \\
\hline Muscle & 12 & 50.82 & 3.015 \\
\hline Visceral fat & 20 & 5.125 & 0.1829 \\
\hline SI wall & 1 & 50.82 & 3.015 \\
\hline SI content & 20 & 51.63 & 4.622 \\
\hline
\end{tabular}

\section{Measurements}

The measurements were conducted in an anechoic chamber in University of Oulu using Agilent VNA 8720ES. The measured frequency band was selected $3.1-10.6 \mathrm{GHz}$, according to IEEE802.15.6 standard for the low-band UWB mandatory channel. We also selected large bandwidth to get good time resolution in the evaluations. Transmitted power was selected to be low due to safety reasons, being only 5 $\mathrm{dBm}$.

The antenna prototypes were connected to the VNA by using $8 \mathrm{~m}$ long Huber+Suhner SUCOFLEX 104PEA measurement cables. A proper calibration was performed before the measurements. The VNA was set to sweep 100 times for each scenario, collecting 1601 frequency points for each measurement set. The measurements were conducted in the frequency domain to obtain radio channel's frequency responses (S21 parameters), which were later transformed into time domain in Matlab using inverse fast fourier transform (IFFT).

In the measurements assisted seven volunteers: three females and four males having different size and body balance. The information about the volunteers is presented in Table II. All the volunteers were not able to assist in all the measurement cases.

Before conducting measurements with the human volunteers, the safety of the measurements were confirmed by simulating Specific Absorption Ratio (SAR) with the voxel models. The SAR value was calculated for $10 \mathrm{~g}$ averages with antenna-skin distance $0 \mathrm{~mm}$, which provides SAR value $0.0036 \mathrm{~mW} / \mathrm{kg}$, whereas the safety limit is 2.0 $\mathrm{W} / \mathrm{kg}$ [15]. Hence, the measurements do not cause risks for the volunteers. SAR values for the layer model is presented in [13]. Moreover, we applied permission for the measurements from the Ethical Committee of University of Oulu, Eudaimonia, which approved our application. 
Table II. Information about volunteers.

\begin{tabular}{|l|l|l|l|l|l|}
\hline Volunteers & Waist & $\begin{array}{l}\text { Fat } \\
\%\end{array}$ & $\begin{array}{l}\text { Viscera } \\
1 \text { fat } \%\end{array}$ & $\begin{array}{l}\text { Muscle } \\
\%\end{array}$ & Age \\
\hline M1 & 82 & 18 & 5 & 39 & 36 \\
\hline M2 & 101 & 23 & 10 & 36 & 45 \\
\hline M3 & 103 & 32 & 14 & 31 & 47 \\
\hline M4 & 101 & 32 & 12 & 30 & 49 \\
\hline F1 & 76 & 30 & 5 & 34 & 41 \\
\hline F2 & 72 & 36 & 4 & 22 & 25 \\
\hline F3 & 71 & 27 & 4 & 35 & 45 \\
\hline
\end{tabular}

\section{Antenna location options}

In this study, we evaluated four different antenna location possibilities. In all the cases, the distance between the antenna-cable and skin is 0mm unless is otherwise stated. Fig.s 4-5a-d present the antenna locations in the measurement and simulation cases. In the first option, presented for the measurement case in Fig 4a and simulation case in $5 \mathrm{a}$, the antennas are located side-by-side on the navel, so the antenna separation distance is $0 \mathrm{~cm}$. The second option, presented in Fig.s 4-5b, is similar to the first option, except the antenna separation distance is $2 \mathrm{~cm}$. The simulation results of these two options are presented in [7], except in this study the simulation model is modified to correspond more realistic case by tilting the antennas along the shape of the abdomen shape.

In the third antenna location option, presented in Fig.s 4$5 \mathrm{c}$, the antennas are located symmetrically respect to the navel, having $8 \mathrm{~cm}$ antenna separation distance. The fourth antenna location option, "navel-flank", presented in Fig 4$5 \mathrm{~d}$, resembles the case where, for instance, the monitoring device is located on the flank and one of the monitoring antennas are located on the middle of the abdomen, i.e, on the navel. In this scenario, the size and body shape of the monitored person has strong impact of the channel characteristics since the distance between the antennas vary significantly depending on the size of the abdomen. For the simulations with the voxel models, this case is challenging due to the position of the hands of the voxel models. If we put the antennas on the similar location as in the measurements, the cavities of the antennas cut the hand, which changes the antenna properties significantly. Thus, we had to locate antennas of the voxel model closer to the abdomen area, which evidently have impact on the channel responses.

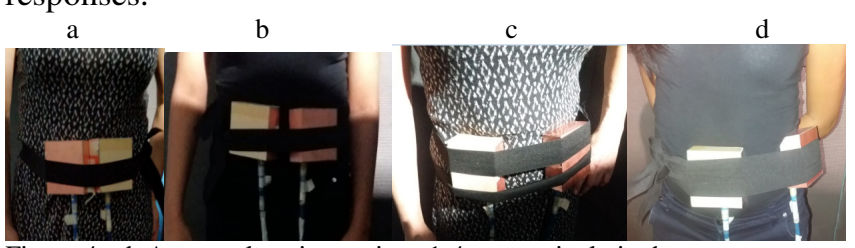

Figure 4a-d. Antenna location options 1-4, respectively in the measurements.

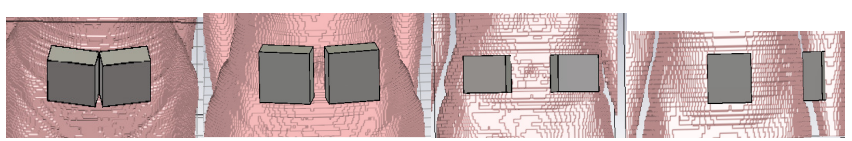

Figure 5a-d. Antenna location options 1-4, respectively in the measurements.

\section{COMPARISONS OF SIMULATION MODELS}

In this section, the IR-simulation results obtained using Laura and Gustav voxel models as well as multilayer models are compared in different antenna locations.

First, we evaluate the antenna location option 1, i.e. antennas are symmetrically respect to the navel with antenna separation distance $0 \mathrm{~cm}$. The impulse responses (IRs) are presented in Fig. 6. In this case, the level of the main peaks obtained with Laura, Gustav, and planar layer models are very similar, only approximately $1 \mathrm{~dB}$ difference is seen. Instead, the difference to bended layer model is obvious, over 5dB. Level of the side peaks in Laura-voxel's and planar multilayer case is surprisingly similar, whereas the level of side peaks of Gustav and bended layer model are more similar to each other, except around $3.5 \mathrm{~ns}$ time instant. The reason for differences between the simulation models is assumed to be due to two different reasons: 1) differences on the surfaces (whether it is smooth or pixelized respect to the antennas) and 2) differences on the antenna tilts. Besides, the pixelized structure due to meshcells of the voxel model can have impact on the results depending how the antennas are located. For instance, although the antenna-cable-skin distance is $0 \mathrm{~cm}$, with voxel models there can be much more air between the antenna and skin than with layer models, as shown in Fig. 7a, which is a cross section of Laura-voxel. This is one reason, why the channel simulated with voxel models is stronger than those simulated with layer models. Besides in certain antenna location cases, simulation of the antenna cable-skin distance $0 \mathrm{~mm}$ is not even possible with these kinds of large cavities and voxel model, as seen from the cross cut of Gustav model in Fig 7b. In this case antenna cable-skin distance is $2 \mathrm{~mm}$, which is minimum possible because the cavity is touching the skin surface on the upper part of the cavity. In the lower part of the cavity there is $4 \mathrm{~mm}$ space between the skin and cavity. Thus, in this case the minimum possible simulated antenna cable-skin distance is $2 \mathrm{~mm}$.

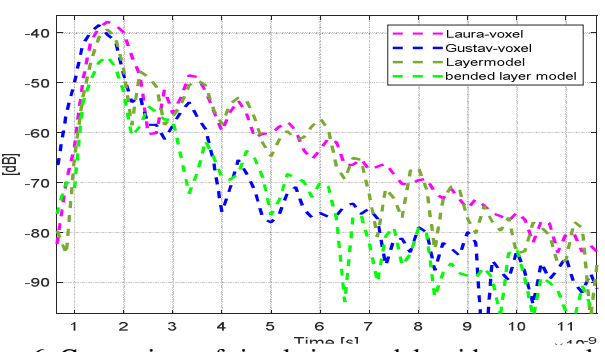

Figure 6. Comparison of simulation models with antenna location option 1.
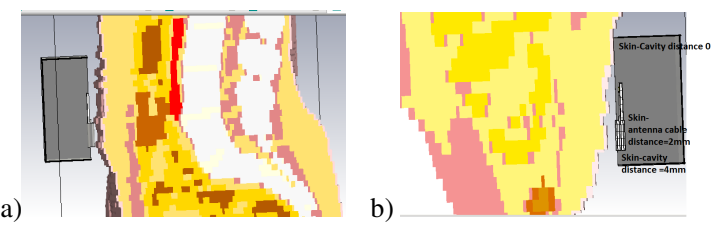

Figure 7. Cross cut of the Gustav-voxel in the antenna location option 2.

Next, the impulse responses obtained with different simulation models are compared in the case of antenna location option 2. In this case, the antenna cable-skin distance 0 is not possible as explained above, and the minimum antenna-cable distance is $2 \mathrm{~mm}$. Thus, we evaluate channels both for $\mathrm{d}=0 \mathrm{~mm}$ and $\mathrm{d}=2 \mathrm{~mm}$ cases. The results are presented in Fig. 8. In this case, the IRs obtained with Gustav-voxel and the layer model at $\mathrm{d}=2 \mathrm{~mm}$ case are quite similar. As $d=0 \mathrm{~mm}$, there is a clear difference between Laura-voxel, planar layer model, and bended layer model. Surprisingly, the difference between the layer model and bended layer model is even $10 \mathrm{~dB}$. Apparently, the curved surface and antenna tilts have impact even at so small antenna distances. 


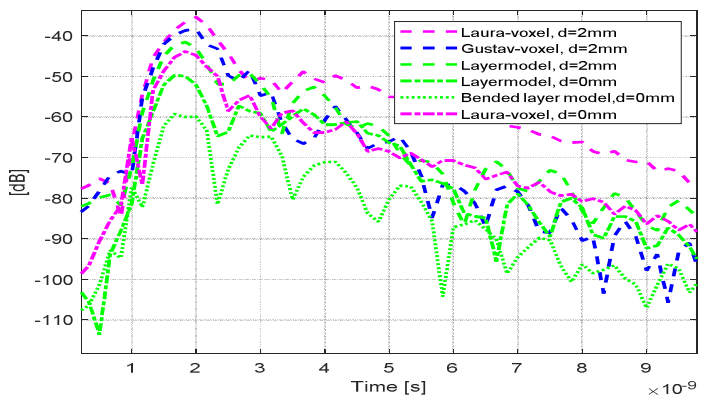

Figure 8. Comparison of simulation models with antenna location option 2.

Next the simulations models are verified in the antenna location option 3 , where antennas are located symmetrically respect to the navel, with separation distance $8 \mathrm{~cm}$. In this case, $d=0$ was not possible with Laura-model (due to unfavorable position of the antennas as explained above) so we included only Gustav-voxel's results. Fig. 9 presents the impulse responses obtained with Gustav-voxel, planar layer model and bended layer model. The results are surprisingly similar: timing and the strength of the main peaks are similar as well as side peak are at the same level until time instant 5 ns. After $5 \mathrm{~ns}$, the side peaks of the planar layer model are significantly higher than those of Gustav-voxel and bended layer model, which is due to different propagation in the planar model than in the curved model.

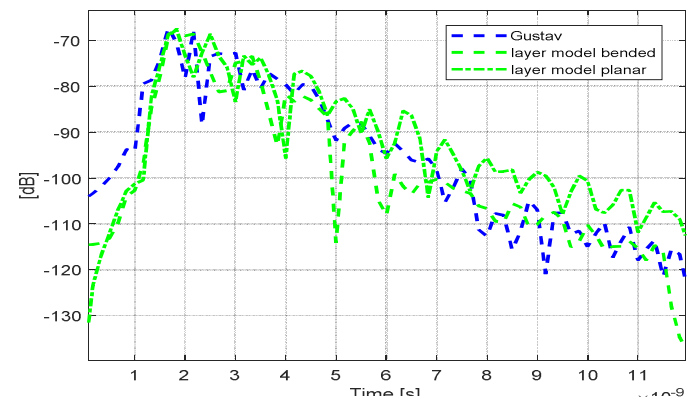

Figure 9. Comparison of simulation models with antenna location option 3.

Next, the simulated impulse responses for the antenna location option 4 are compared. For this case, we can choose only Gustav-voxel and the bended layer model. The problem with Laura-voxel is the position of the hand, which prevents setting the cavity-backed antenna on the side properly, because edges of the cavity cut the hips or the hand. Also with Gustav-model, the hand prevent to put the antenna completely on the other side, as presented in Fig. 10, as well as in this case $d=4 \mathrm{~mm}$ is the only possibility due to shape of the voxel, as explained above. The impulse responses obtained with Gustav-voxel and the bended layer model are shown in Fig. 11. One can see clear difference between these results, which is obvious due to different antenna-cable-skin distances.

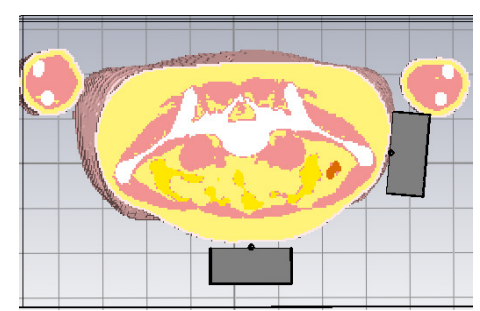

Figure 10. Cross-cut of Gustav-voxel in the antenna location option 4.

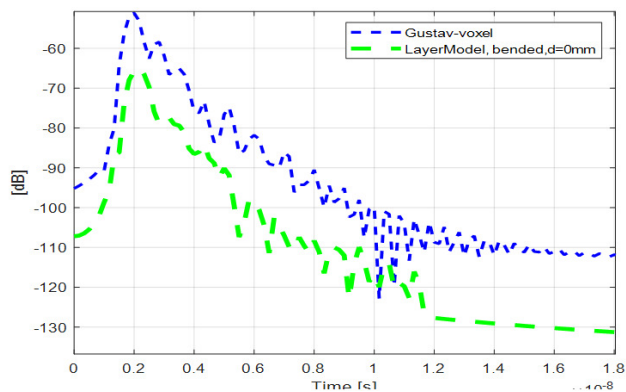

Figure 11. Comparison of simulation models with antenna location option 4

\section{MEASUREMENT AND SIMULATION RESULTS}

In this section, we study time domain channel characteristics on the abdomen area with measurement and simulations results. For the clarity of the figures, we have separated male's and female's cases in different figures in the cases where we have measurement data for several volunteers.

\section{A. Antenna location 1, side-by-side case}

Fig.s 12a-b present the measurement results with the antenna location option 1 for female volunteers and for male volunteer, respectively. The impulse responses obtained with the simulation models are included in the figure for the comparison. It is found, that in females' and males' cases there is only negligible difference between the volunteers' impulse responses. This is due to the fact, that since antennas are so close to each other, the size and body shape of the volunteer do not have so much impact on the on-body channel characteristics.

As we compare the measurement and simulation results, we can see a clear difference in the levels of the main peaks. With females, the difference is around $5 \mathrm{~dB}$, when compared to IR obtained with the bended layer model, whereas the difference is over $10 \mathrm{~dB}$ when compared to the voxel model results. With male volunteers, these differences are slightly higher.

There are several reason for differences between the simulated and measured results. First is related to the uncertainities always present in the measurements, such as differences between the antenna properties of the prototype and the simulation model, cabling, inaccurate position of the antennas in the measurements, etc. For instance even small antenna tilt angle may have several $\mathrm{dBs}$ difference in the channel response. Especially in the abdomen area, tilt angles may vary significantly depending on the shape of the abdomen area. Furthermore, as presented in [8], the impact of antenna and body distance can have significant impact on the channel characteristics. Simulation based studies of [8] show that only $2 \mathrm{~mm}$ difference can cause even $8 \mathrm{~dB}$ difference to the level of the main peaks. Hence, the thickness of the cloth may affect significantly on the measurement results.

\section{B. Antenna location option 2: Antenna distance $2 \mathrm{~cm}$}

Next, the channel characteristics are evaluated with the $2 \mathrm{~cm}$ separation between the antennas. The measured impulse responses of the female and male volunteers are presented in Fig.s 13a-b, respectively. The impulse responses obtained with Laura-voxel and the bended layer model are included as the reference. Gustav-voxel results was not included, since $d=0 \mathrm{~mm}$ case could not be provided by the Gustavvoxel in this antenna location case as explained earlier. As 
one can note, now we see slightly more difference in the impulse responses of the volunteers with different sizes. There is more variation on the level of the peaks between different volunteers. The differences are assumed to be due to different tilt angles of the antennas: for the lean volunteers with "straight" abdomen area, the tilt is minor and those "more curved" abdomen area. For instance, the impulse responses of the leanest volunteers (F3 and M1) drop more quickly than for the others.

Next, the measured results are compared with the simulation results. Interestingly, in this antenna location case, the main peak of the bended layer model is close to those of F3 and M1. In the case of female volunteers, the level of bended model's side peaks are at clearly higher level than those of the volunteers at time period 2.75-4.2 ns. Instead, for the male volunteers, there is clear difference between the side peaks of the bended layer model and volunteers only around $4 \mathrm{~ns}$.
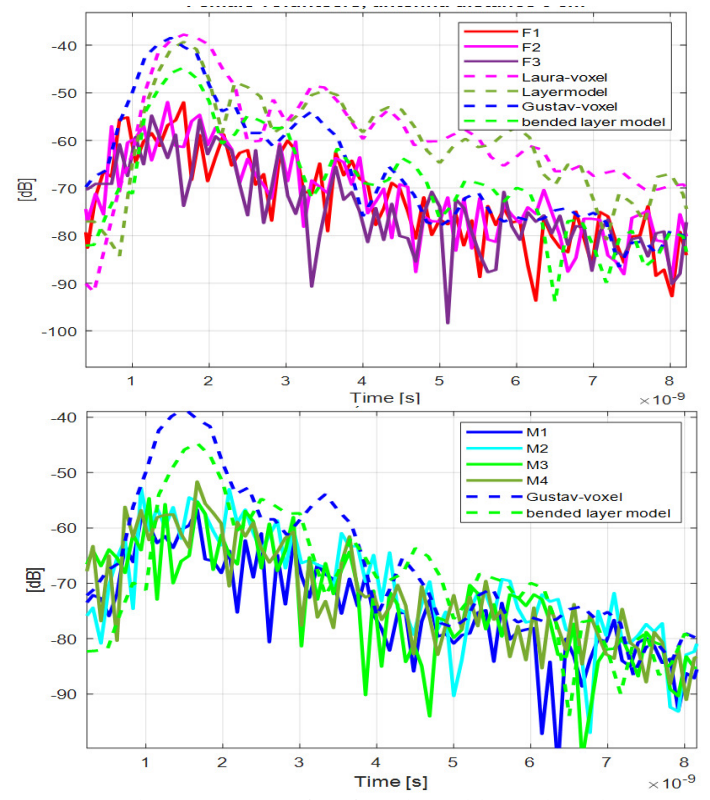

Figure 12. Impulse responses of a) female and b) male volunteers compared with the simulation results in the case of antenna location option 1.
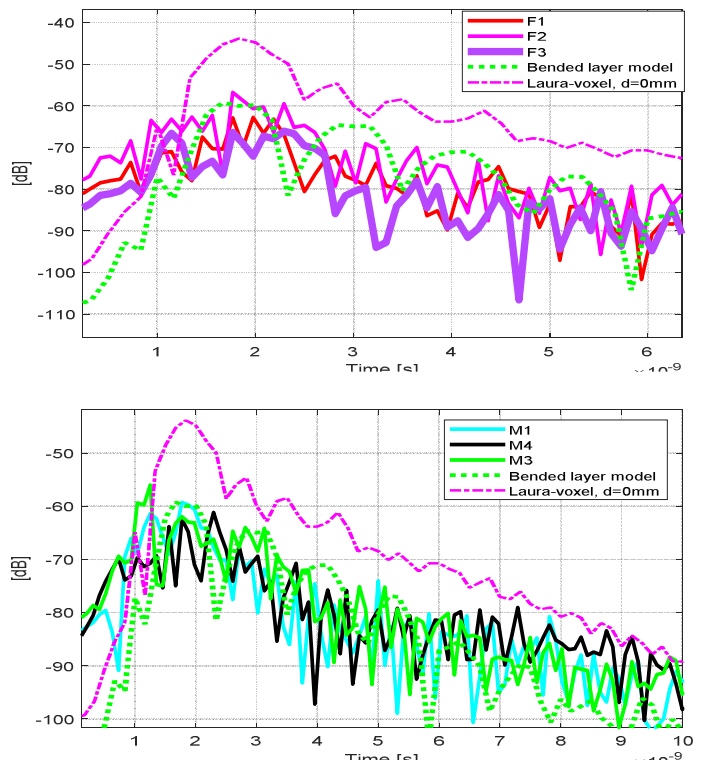

Figure 13. Impulse responses of the a) female and b) male volunteers compared with the simulation results in the case of antenna location option 2

\section{Antenna location option 3: Antenna distance $8 \mathrm{~cm}$}

Next, the channel characteristics are evaluated as the antenna separation distance is $8 \mathrm{~cm}$. Only volunteers F1 and M4 participated on this study case. The measured and simulated impulse responses are shown in Fig. 14. One can see clear difference in the impulse responses of two volunteers. Interestingly, M4's main peak is at the same level as those of the bended layer model and Gustav-voxel model but there is a clear difference in the shape of the main peaks. Instead, the shape of the main peak of F1 is similar to those of the layer model but there is a clear level difference, almost $10 \mathrm{~dB}$. Side peaks are at the same level starting from $3 \mathrm{~ns}$ onwards, but there is a clear difference in the side peaks at time period 2.5ns-3ns. Again, the differences between the impulse responses of volunteers F1 and M4 are assumed to be due to different antenna tilts, since the shape of the abdomen area is different for these volunteers. Besides, at this antenna separation distance the propagation through the tissues can have a clear impact. For instance, different amount of fat on the abdomen area may cause some differences.

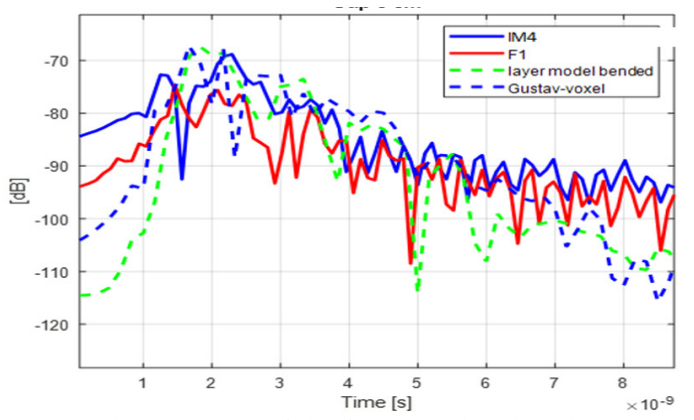

Figure 14. Impulse responses of the female and male volunteers compared with the simulation results in the case of antenna location option 4.

\section{Antenna location option 4: Navel-flank case}

For the antenna location option 4, the measured impulse responses are presented in Fig. 15, in which the simulation results are included in for the comparison. In this case, we can see significant differences between the volunteers. One clear reason for the differences is the position of the antenna on the flank: in the measurements it was challenging to get the antenna attached on the flank easily and hold it there in the same place. It easily moved towards the navel causing the antenna separation distance getting smaller. This can be seen as different timing and strengths of the main peaks between the volunteers. The case of volunteer F2 describes well this situation: the main peak arrives $0.5 \mathrm{~ns}$ earlier compared the other volunteer's case because antennas are closer to each other, and thus, the strength of the channel is also clearly higher. This can be validated from Figure $7 \mathrm{a}$, where the it can be seen that the second antenna is not on the side as it is supposed to be but it has slightly moved towards the antenna located on the navel.

In order to verify impact of the slight transfer from the other flank towards, we study volunteer M3's case as the antenna separation distance is same as that of F2. The results are shown in Fig. 16. As we can see, the impulse responses of volunteers F2 and M3 are very similar now as the antenna separation distance is fixed to be same. This proves how much the antenna separation distance has impact especially in this kind of more challenging antenna location option in terms of propagation. 


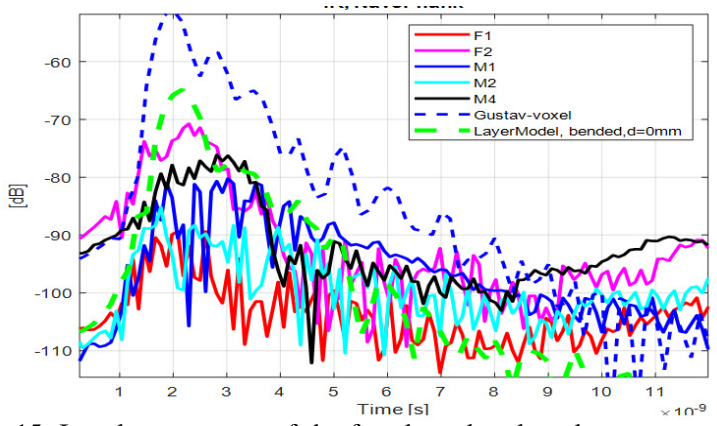

Figure 15. Impulse responses of the female and male volunteers compared with the simulation results in the case of antenna location option 4.

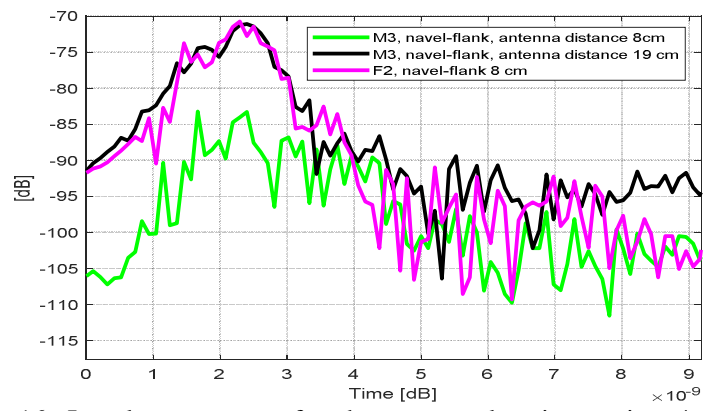

Figure 16. Impulse responses for the antenna location option 4 as the antenna separation distance is $8 \mathrm{~cm}$ and $19 \mathrm{~cm}$ for the volunteer M3. Impulse response of the volunteer $\mathrm{F} 2$ as a reference.

\section{CONCLUSIONS AND FUTURE WORK}

This paper presented a comprehensive simulation and measurement data -based study on the UWB on-body channel characteristics on the human abdomen area. For the simulations, we used two voxel models and two layer models. Measured data consists of channel characteristics of seven volunteers having different body size and body balance. The simulated and measured data was compared and reason for differences was discussed.

It was found that the both voxel models and layer models have their pros and cons. The main advantage of the voxel models is that they are anatomically similar to a human body which enables the more realistic evaluation of the channel characteristics than with layer models, especially in the inbody communications. Due to this, the shape of the impulse responses simulated with the voxel models correspond more to the realistic case than those of the layer models. However, the pixelized surface of the voxel tissues may affect significantly on the on-body channel characteristics. As it was shown in the paper, the pixelized surface may increase the amount of air between the antenna and the skin, which enhances the channel strength. Besides, in all the cases it was not even possible to set the antenna with such a large cavity on the voxel model's skin surface so that distance between the antenna cable and skin was $0 \mathrm{~mm}$. For these reasons in several antenna location options, the channels simulated with the voxel models were stronger than those simulated with layer models. These drawbacks may be avoided if antennas without large cavities is used, which is one of our future work plans. Besides, the use of voxel models with smaller pixel size may also alleviate this problem. The main advantages of the layer models are the smooth surface corresponding more the real skin surface, as well as flexibility of the model size. One can easily increase or decrease the size of the model and hence get channel characteristics corresponding to a smaller or larger person.

From the studies with the measurement data, it was found that the channel characteristics between the volunteers having different body size vary clearly in the cases that antenna separation distance is larger. With smaller antenna distances, the difference is minor.

As a future work, besides of the above-mentioned studies with smaller antennas and voxel models with higher resolution, our plan is conduct simulations with other voxel models as well as conduct more measurements in different antenna location options.

\section{ACKNOWLEDGMENT}

This research has been financially supported by the project WBAN Communications in the Congested Environments and in part by Academy of Finland 6Genesis Flagship (grant 318927). We would like to thank all the volunteers who participated in the measurements. Ilkka Virtanen, Timo Mäkinen, and Jari Sillanpää from University of Oulu deserve acknowledgement for their help to enable the exhaustive simulations. Furthermore, Dr. Markus Berg from CWC is acknowledged for his advices with the CST simulator.

\section{REFERENCES}

[1] P. Turalchuk, I. Munina, V. Pleskachev, V. Kirillov, O. Vendik, I. Vendik, "In-Body and On-Body Wave Propagation: Modeling and Measurements," iWAT conference, 2017.

[2] P. A. Floor, R. Chavez-Santiago, S. Brovoll, O. Aardal, J. Bergsland, O-J. H. N. Grymyr, P. Halvorsen, R. Palomar, D. Plettemeier, S-E. Hamran, T. Ramstad, I. Balasingham, "In-Body to On-Body Ultrawideband Propagation Model Derived from Measurement in Living Animals," IEEE Journal of Biomedical and Health Informatics, 2015.

[3] R. Chavez-Santiago, J. Wang, I. Balasinham, "The Ultra Wideband Capsule Endoscope," Internatiomal Conf. on Ultra Wideband, 2013.

[4] S. Stoa, R. Chavez-Santiago, I. Balasinham, "An ultra wideband communication channel model for capsule endopscopy," ISABEL2014

[5] P. Ara, K. Yu, S. Cheng, E. Dutkiewicz, M. C. Heimlich, "Human Abdomen Path-Loss Modeling and Location Estimation of Wireless Capsule Endoscope Using Round-Trip Propagation Loss," IEEE Sensor Journal, Vol. 18, No. 8, 2018.

[6] K. M.S. Thotahewa, J-M Redoute, M. R. Yuce, "Electromagnetic Power Absorption of the Human Abdomen from IR-UWB Based Wireless Capsule Endoscopy Devices," ICUWB, 2013.

[7] M. Särestöniemi, C. Kissi, C. Pomalaza Raez. M. Hämäläinen, J. linatti "Propagation and UWB channel characteristics on human abdomen area," EUCAP2019, 2019.

[8] M. Särestöniemi, C. Kissi, C. Pomalaza Raez. M. Hämäläinen, J. Iinatti "Impact of the Antenna-Body Distance on the UWB On-Body Channel Characteristics," ISMICT2019, 2019.

[9] K. Lopez, A. Thielesn, G. Vermeeren, W. Joseph, "Characterization of Path Loss and Absorption for a Wireless Frequency Link Between an In-Body Endoscopy Capsule and A Receiver Outside the Body,"EURASIP 2014.

[10] N. Dey; A. S. Ashour; F. Shi; and R. S. Sherratt, "Wireless Capsule Gastrointestinal Endoscopy: Direction-of-Arrival Estimation Based Localization Survey", IEEE Reviews in Biomedical Engineering, Vol. 10, pp. 2 - 11, 2017.

[11] H. Mateen; R. Basar; A. U. Ahmed; M. Y. Ahmad, "Localization of Wireless Capsule Endoscope: A Systematic Review", IEEE Sensors Journal, Vol. 17, pp. 1197 - 1206, 2017.

[12] C. Kissi; M. Särestöniemi; C. Pomalaza-Raez; M. Sonkki; and M. N. Srifi, "Low-UWB directive antenna for Wireless Capsule Endoscopy localization", BodyNets2018 conference, Oulu, Finland, October 2018.

[13] C. Kissi; M. Särestöniemi; T. Kumpuniemi, M. Sonkki, S. Myllymäki, M. N. Scrifi, C. Pomalaza-Raez; "Low-UWB antennas in the Vicinity of Human Body," submitted to ISMICT 2019 conference.

[14] CST Microwave Studio, [Online]. Available: http://www.cst.com https://www.icnirp.org/cms/upload/publications/ICNIRPemfgdl.pdf 